\title{
FIOCRUZ E A SOCIEDADE: uma visão da divulgação em jornais e revistas a partir da base de Currículos Lattes
}

\author{
FIOCRUZ AND A SOCIETY: \\ $A$ vision of magazine and magazine dissemination based on Lattes Curricula
}

\author{
Rosangela Cordeiro de Souza Assef Neto \\ Maria Cristina Soares Guimarães ${ }^{2}$ \\ Cícera Henrique da Silva ${ }^{3}$ \\ Rosane Abdala Lins ${ }^{4}$
}

\begin{abstract}
RESUMO
Na relação entre ciência e sociedade e o papel do pesquisador, bem como 0 das instituições de pesquisa são fundamentais no processo de divulgação da ciência. Este artigo traz o resultado de uma pesquisa que utilizou o Curriculum Lattes como fonte de dados, com o objetivo de mostrar quais canais utilizados por uma instituição de pesquisa no diálogo com a sociedade. São apresentados os principais canais de comunicação e como essa se deu em um período de 15 anos. Entre os canais de comunicação utilizados pela comunidade de pesquisadores, um deles é uma revista de divulgação científica que representa um papel real nesse processo, pois é vendida em bancas de jornais. Com a promoção da divulgação de resultados de pesquisa para sociedade, a comunidade científica exerce um papel fundamental para a reflexão sobre os impactos sociais e culturais das descobertas da ciência.
\end{abstract}

Palavras-chave: Divulgação científica. Ciência e sociedade. História da divulgação científica.

\section{ABSTRACT}

The relationship between science and society, the role of the researcher, as well as the research culture are fundamental in the process of science dissemination. This article presents the result of a research that uses the Lattes Curriculum as a data source, aiming to show the links to a research without dialogue with society. The main channels of communication are promoted and as it happened in a period of 15 years. Researchers' media in the research community is a science journal that represents a real process in this sense, as it is sold at newsstands. By promoting the dissemination of results to society, the scientific community plays a key role in reflecting on the social and cultural aspects of science's discoveries.

Keywords: Scientific dissemination. Science and society. History of scientific dissemination.

Artigo recebido em 28/03/2019 e aceito para publicação em 15/05/2019.

1 Mestranda em Ciência da Informação e Assistente de Pesquisa dn Laboratório de Informação Científica e Tecnológica em Saúde do Instituto de Informação e Comunicação Científica e Tecnológica em Saúde da Fundação Oswaldo Cruz, Brasil. E-mail: rosangela. cordeiro@icict.fiocruz.br.

2 Doutora em Ciência da Informação pelo convênio entre o Instituto Brasileiro de Informação em Ciência e Tecnologia e a Universidade Federal do Rio de Janeiro, Brasil. Pesquisadora Sênior do Laboratório de Informação Científica e Tecnológica em Saúde do Instituto de Informação e Comunicação Científica e Tecnológica em Saúde da Fundação Oswaldo Cruz, Brasil. E-mail: cristina.guimaraes@ icict.fiocruz.br.

3 Doutora em Sciences de I Information Et de La Communication pelo Université Paul Cézanne Aix Marseille III, França. Pesquisadora Sênior do Laboratório de Informação Científica e Tecnológica em Saúde do Instituto de Informação e Comunicação Científica e Tecnológica em Saúde da Fundação Oswaldo Cruz, Brasil. E-mail: cicera.henrique@icict.fiocruz.br.

4 Doutora em Informação e Comunicação em Saúde pela Fundação Oswaldo Cruz, Brasil. Pesquisadora do Laboratório de Informação Científica e Tecnológica em Saúde do Instituto de Informação e Comunicação Científica e Tecnológica em Saúde da Fundação Oswaldo Cruz, Brasil. 


\section{INTRODUÇÃO}

Comunicação na etimologia da palavra, deriva do termo latino communicare, que significa "partilhar, participar algo, tornar comum". A comunicação científica faz parte do fazer ciência, visa, basicamente a disseminação da informação entre os pares, diz respeito à transferência de informação científica, tecnológica ou associada a inovação, destinadas aos especialistas em determinadas áreas do conhecimento. Quando o pesquisador, ao cumprir a missão de dar satisfação à sociedade se dirige diretamente a esta, o processo recebe a denominação de divulgação científica, que trata da utilização de recursos, técnicas, processos e produtos (canais) para a veiculação de informação científica, tecnológica ou inovação para o público leigo (BUENO, 2010). Por conseguinte, os veículos ou canais utilizados neste processo são chamados de divulgação científica.

Enquanto um bem público, a ciência deve estar comprometida em contribuir com a circulação de ideias e resultados de pesquisa, como um exercício de reflexão sobre os impactos sociais e culturais das descobertas do cientista. Na relação entre ciência e sociedade, o papel do cientista é colocado ao lado da instituição de pesquisa, como responsáveis por promover a divulgação da ciência, ação fundamental para o próprio avanço da ciência (CANDOTTI, 2014).

0 processo de comunicação da informação científica à sociedade, em todos os campos do saber, tem sido colocado como ponto de discussão no processo de como comunicar a ciência. É imperativo buscar pontos de conexão entre ciência e sociedade, para isso temas como disseminação da informação, divulgação científica, jornalismo científico, acolhem um conjunto diferenciado de metodologias, práticas, instrumentos e abordagens, compartilhando o mesmo objetivo de promover a comunicação científica (GUIMARÃES, 2014).

Para os cientistas e para os que trabalham em instituições de pesquisa, a divulgação científica pode ser considerada uma atividade de prestação de contas à sociedade, diante do investimento público feito para a pesquisa, mesmo que de forma indireta.

A Fundação Oswaldo Cruz (Fiocruz), considerada a maior instituição de pesquisa em saúde do Brasil, possui em seu corpo de trabalho pesquisadores, cientistas que buscam por meio de suas pesquisas encontrar respostas às diversas questões de saúde. Vinculada ao Ministério da Saúde, a Fundação Oswaldo Cruz (Fiocruz) é considerada a maior instituição de ciência e tecnologia em saúde da América Latina, onde são desenvolvidos mais de mil projetos de pesquisa e desenvolvimento tecnológico que produzem conhecimento para o controle de doenças como aids, malária, chagas, tuberculose, entre 
outras, além de temas ligados à saúde coletiva. É a principal instituição não-universitária de formação e qualificação de recursos humanos para o Sistema Único de Saúde (SUS), além de possuir diversos programas de pós-graduação stricto sensu e lato sensu (Fiocruz, [s.d.]).

Com presença em todas as regiões do Brasil, possui 16 unidades técnico-científicas e um escritório em Maputo, capital de Moçambique. As unidades estão voltadas para o ensino, pesquisa, inovação, assistência, desenvolvimento tecnológico e extensão no âmbito da saúde.

Visando entender de que forma se dá o diálogo entre ciência e sociedade e na qualidade de pesquisadores especializados em estudos de informação, as questões que se colocam no presente estudo são: Quais os tipos de canais mais utilizados para concretização da comunicação da Fiocruz com a sociedade? Como tem sido essa comunicação ao longo dos anos?

A pesquisa foi realizada a partir de dados da base de Curriculo Lattes $^{5}$, onde foram coletados os dados produzidos pelo quadro de servidores da Fiocruz.

\section{A DIVULGAÇÃO CIENTÍFICA NO BRASIL}

A divulgação científica no Brasil tem um histórico de no mínimo 200 anos, apesar de ter sido feita de forma incipiente nesse período. Tem atendido ao longo desse período a motivações diversas, apresentando características que refletiam as necessidades e o contexto de cada momento.

Até o início do século XIX, a prática científica ou mesmo divulgação de ideias científicas no país era praticamente inexistente. Somente com a chegada da Corte portuguesa no país, no início do século XIX, com a suspensão da proibição de imprimir, com a criação da Imprensa Régia, em 1810, os poucos brasileiros que foram estudar na Europa, ao retornar para o brasil no final do século XVIII e início do século XIX, tiveram espaço para contribuir para uma difusão, ainda que tímida, da ciência. Em pesquisa divulgada em 2002, Moreira e Massarani concluíram que durante o século XIX, surgiram cerca de 7.000 periódicos no Brasil, dentre estes, aproximadamente 300 relacionados à ciência, produzidos por instituições ou associações científicas. Alguns destaques desse período trazidos pelos autores são a Revista Rio de Janeiro, de 1876 e a Ciência para o povo de 1881, ambas com textos de divulgação científica, com assuntos relacionados a febre amarela, cérebro, saúde e comportamento, sonambulismo, entre outros (MASSARANI; MOREIRA; BRITO, 2002).

5 http://lattes.cnpq.br/. A plataforma de Currículo Lattes é a base de dados de currículos de todos os estudantes e pesquisadores do Brasil. 
As atividades de divulgação científica em todo o mundo aumentaram em decorrência da Segunda Revolução Industrial na Europa evidenciadas pela realização das Exposições Universais que percorreram o mundo a partir de 1851. No Brasil, deu-se início às Exposições Nacionais em 1861 onde a primeira delas teve um número de visitantes superior a 47.000 pessoas. Essa exposição abriu portas para a participação do país nas Exposições Universais, o que ocorreu entre 1862 e 1889 (MASSARANI; MOREIRA; BRITO, 2002).

Ainda nesse período, iniciaram-se as Conferências Populares da Glória, uma iniciativa que, de acordo com o seu criador, tinha como principal objetivo a instrução do povo. Os protagonistas das conferências acreditavam que com a divulgação da ciência e da cultura, poderia se mudar o país. Foi uma iniciativa que perdurou por mais de 20 anos, mesmo notando-se a tendência à participação da aristocracia:

[...] através de relatos da época, constatamos que a sua plateia era constituída por um seleto público, sendo notada a presença da família imperial, da aristocracia da corte, de profissionais liberais e estudantes (FONSECA, 1996, p. 2).

Um destaque que deve ser dado às Conferências Populares da Glóriaé a temática abordada nesses encontros, que abrangia desde temas culturais até ao conhecimento das ciências como matemática, biologia, medicina, etc. Essas conferências eram noticiadas nos jornais mais importantes da época como: Jornal do Comércio, Gazeta de Notícias e Diário do Rio de Janeiro, onde estes publicavam não somente a realização das conferências, como muitas vezes o resumo do que era apresentado (FONSECA, 1996).

Na última década do século XIX ocorreu uma diminuição das principais atividades de divulgação científica, bem como o número de revistas e artigos com essa finalidade, o que também aconteceu no contexto internacional (MASSARANI; MOREIRA; BRITO, 2002).

No início do século XX, foi criada a Sociedade Brasileira de Ciências, que em 1922 transformouse em Academia Brasileira de Ciências ( $A B C)$. Foi a partir da $A B C$, com a iniciativa de alguns membros e sócios, como o antropólogo Edgard Roquette-Pinto, que foi criada a Rádio Sociedade do Rio de

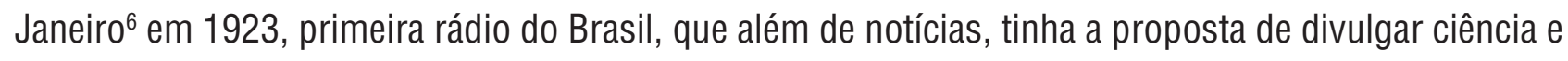
cultura para a população. A rádio exerceu então um importante papel nesse tempo de popularização da ciência, através de suas transmissões. Em 1936, ela foi doada ao governo brasileiro, e passou a chamar-

6 http://www.fiocruz.br/radiosociedade/cgi/cgilua.exe/sys/start.htm?sid=2 
se Rádio MEC (Ministério da Educação e Cultura), hoje ligada à Empresa Brasileira de Comunicação - EBC ("Rádio Sociedade", [s.d.]).

Ao longo da década de 1920, houve intensificação da divulgação da ciência, com o surgimento de revistas e boletins com espaço para divulgação científica, os jornais de maior ou menor circulação também abriram espaço para divulgação de notícias como a vinda de cientistas famosos ao Brasil, como Marie Curie e Einstein. Nesse período também foram publicados alguns livros voltados para a divulgação da ciência, como o Neo-relativismo Eeinsteiniano, de Carlos Penna Botto e Conceito atual de vida, de Roquette-Pinto. Também foram realizadas conferências públicas relacionadas à ciência, como as conferências públicas realizadas pela ABC (MASSARANI; MOREIRA; BRITO, 2002).

Durante os anos de 1930 a 1970, o Instituto Nacional do Cinema Educativo (INCE)7 produziu centenas de filmes curtos (com duração de até 30 minutos) com o propósito de divulgar temas científicos e tecnológicos e divulgar as instituições científicas do país (MASSARANI; MOREIRA; BRITO, 2002).

Refletindo, entre outras coisas, o reconhecimento e a institucionalização da ciência no Brasil, em 1948 foi criada a Sociedade Brasileira para o Progresso da Ciência (SBPC), nos moldes das que já existiam em outros países. Pouco tempo depois da criação da SBPC, foram criados em 1951 pelo governo federal o Conselho Nacional de Desenvolvimento Científico e Tecnológico (CNPq) e a Coordenação de Aperfeiçoamento de Pessoal de Nível Superior (Capes).

A partir dos anos 1980 surgiram iniciativas que permanecem até o momento, como o programas de divulgação científica na TV, Globo Ciência sas revistas da SBPC, que hoje são editadas pelo Instituto Ciência Hoje (ICH), de cunho específico de divulgação científica, como Ciência Hoje e Ciência Hoje das Crianças, e outras revistas de circulação nacional, mais populares, como as revistas Superinteressante e Galileu.

Além das revistas, os jornais de grande circulação no país criaram seções de ciência, o que é presente hoje em seções conjuntas como ciência e tecnologia, ciência e saúde.

As instituições de pesquisa e universidades também criaram canais de comunicação com a sociedade e com a popularização da internet, viu-se também a criação de sites e portais dessas instituições voltados para divulgação científica.

Seguindo esta tendência, a Fundação Oswaldo Cruz possui hoje alguns canais de comunicação com o público, tais como:

$7 \quad$ Órgão financiado pelo governo de Getúlio Vargas, criado por Roquette-Pinto em 1936. 
- $\quad$ Portal da Fiocruz, um portal com o objetivo de divulgar a instituição, as ações de pesquisa, ensino e desenvolvimento realizadas na instituição e que recebe hoje cerca de 200 mil acessos / mês (FIOCRUZ, [s.d.]);

- Museu da Vida, fundado em 1999 que possui atividades de visita guiada, atividades de educação com cursos de especialização lato e stricto sensu, pesquisa, coleta e preservação de peça museológicas e acervo bibliográfico e exposições itinerantes com o caminhão do Ciência Móvel (“o que é o museu da vida?", [s.d.]);

- $\quad$ Canal Saúde, uma emissora de televisão pública no âmbito do Ministério da Saúde, sediada na própria fundação, que tem como missão produzir conteúdo sobre saúde, os programas exibidos pelo Canal Saúde têm caráter jornalístico e informativo, em concordância com o que preconiza o Sistema Único de Saúde (SUS) (FIOCRUZ, [s.d.]);

- Vídeo Saúde Distribuidora da Fiocruz é um polo de guarda, produção e disseminação de materiais audiovisuais em saúde. Atua na pesquisa, captação, catalogação, produção, fomento e distribuição de produtos audiovisuais (FIOCRUZ, [s.d.]);

- Revista RADIS Comunicação e Saúde, revista voltada para o jornalismo crítico e independente em saúde pública, com o objetivo incluído nas iniciais do próprio nome: reunião, análise e difusão de informação sobre saúde. Em mídia impressa e digital, a revista vem aumentando a interlocução com a sociedade e a voz dos movimentos organizados, sendo enviada para mais de 100 mil assinantes em todos os municípios do país (Radis - comunicação em saúde, [s.d.]).

\section{METODOLOGIA}

Para atender o objetivo desta pesquisa, qual seja o de identificar como ocorre a comunicação da Fiocruz para a sociedade e quais são os principais canais de divulgação científica utilizados por seus pesquisadores, são descritos a seguir os procedimentos metodológicos realizados.

Os dados foram obtidos a partir da extração da produção atribuída aos servidores da Fiocruz a partir do CV Lattes. A Plataforma Lattes é uma iniciativa do Conselho Nacional de Desenvolvimento Científico e Tecnológico (CNPq) para integração de bases de dados de Currículos, de Grupos de Pesquisa e de Instituições em um único Sistema de Informação. 0 Currículo Lattes se tornou um padrão nacional no registro da vida pregressa e atual de pesquisadores e estudantes do país, atualmente considerado 
um padrão brasileiro de avaliação para os órgãos de fomento e utilizada pela maioria das instituições ("Plataforma Lattes CNPq - sobre", [s.d.]).

Dentro das categorias existentes no CV Lattes, foi selecionada a categoria “produções”, seguida de "produção bibliográfica" e finalmente, foram coletados todos os dados existentes na categoria "textos em jornais e revistas" para efeitos desse estudo. Foi feito um recorte temporal para abranger a produção no período 2000 a 2015. De um total de 97.810 itens considerados pelos declarantes como produção bibliográfica, foi selecionada a tipologia explicitada "texto em jornal ou revista", totalizando 3.115 registros. Os dados desta tipologia foram exportados para uma planilha Excel e feita a identificação dos seguintes dados: nome do autor, título do artigo, título da publicação e data.

A partir da análise do título da publicação, foi feita sua categorização em jornal, rádio/tv e revistas.

Ao analisar o total de registros existentes na categoria "revistas", foram identificados registros publicados em periódicos científicos. Diante disto, foi feita a conferência de todos os registros existentes, de forma a identificar se o periódico era científico ou não. Para auxiliar neste processo foi utilizado o portal Ulrichweb8 ${ }^{8}$, além do acesso à página da revista, onde foram considerados a presença de dois ou mais dos seguintes parâmetros: critério para submissão de trabalhos, revisão por pares, periodicidade e ISSN. Os registros publicados em periódicos científicos foram desconsiderados das análises seguintes.

A categoria revistas foi subdividida nas seguintes subcategorias:

1. Revistas de associação de classe/segmento (p.e. Revista da Sociedade Brasileira de Endocrinologia)

2. Revistas de Divulgação Científica

3. Revistas de interesse geral

4. Periódicos científicos

$8 \quad$ ulrichsweb.serialssolutions.com. Portal Não seria uma base de dados cadastral? onde estão registrados mais de 300.000 periódicos ativos, mantida pelo Cambridge Information Group. Neste portal, existe uma ficha catalográfica do periódico, com a indicação do tipo de periódico e se este é científico ou news paper. Além do acesso à página da revista, onde foram considerados, revisão por pares, periodicidade, issn. 


\section{RESULTADOS E DISCUSSÃO}

Do total de 3.115 registros, não foi possível identificar a tipologia de 4 registros. Os 3.111 restantes foram classificados nas categorias demonstradas abaixo:

Tabela 1: Produção em Jornais e Revistas por categoria - período 2000 a 2015

\begin{tabular}{ccc}
\hline Categoria & $\mathbf{N}^{\mathbf{0}}$ & $\%$ \\
\hline Revistas & 2.200 & 70,72 \\
Jornais & 890 & 28,61 \\
Rádio / TV & 21 & 00,67 \\
\hline TOTAL & $\mathbf{3 . 1 1 1}$ & $\mathbf{1 0 0 , 0 0}$ \\
\hline
\end{tabular}

Fonte: elaboração própria a partir dos dados coletados na plataforma Lattes

Observa-se que na categoria "jornais e revistas" existente no CV Lattes, esta também abrange as produções realizada para a Rádio e TV, apesar de ser em uma proporção bem pequena.

A seguir são apresentados os resultados existentes na categoria "revistas", que do total de 2.200 registros, foram excluídos 176 (6\%) registros publicados em periódicos científicos, totalizando 2.024 registros. Os dados existentes nessa categoria estão demonstrados na Tabela 2:

Tabela 2: Produção em Revistas por categoria - período 2000 a 2015

\begin{tabular}{lrc}
\hline Subcategorias & Quant. & $\%$ \\
\hline Revistas de divulgação científica & 1.002 & 50 \\
Revistas de assoc. de classe/segmento & 658 & 32 \\
Revistas de interesse geral & 364 & 18 \\
\hline TOTAL & $\mathbf{2 . 0 2 4}$ & $\mathbf{1 0 0}$
\end{tabular}

Fonte: elaboração própria a partir dos dados coletados na plataforma Lattes

Observa-se nessa tabela, a existência ainda que em número pequeno, de publicações em revistas de interesse geral, que apesar de não serem específicas de divulgação científica, trazem matérias sobre temas relativos à ciência. 
0 gráfico a seguir mostra a evolução da produção total na categoria "jornais e revistas" no período de 2000 a 2015.

Gráfico 1: Produção em jornais e revistas no período de 2000 a 2015

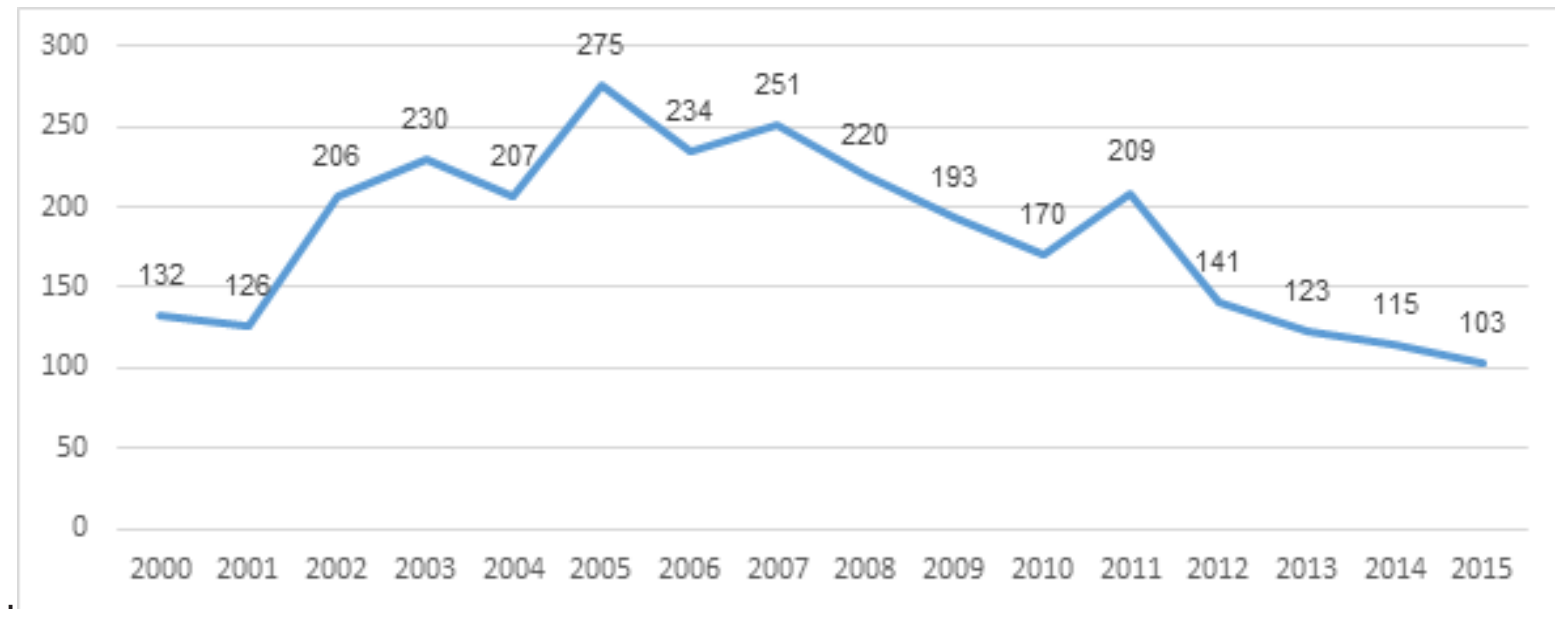

Fonte: Elaboração própria a partir dos dados coletados na plataforma Lattes

É curioso observar 0 decréscimo ocorrido a partir de 2011, sendo menor que a produção registrada no primeiro ano da série (2000) quando de fato a própria Capes passou a valorizar a divulgação científica, apesar de a maioria dos registros estar duplicado na categoria "Democratização/Popularização da ciência" do CV Lattes. No gráfico 2, a seguir apresenta-se a evolução por categoria

Gráfico 2: Produção em jornais e revistas, por categoria no período de 2000 a 2015

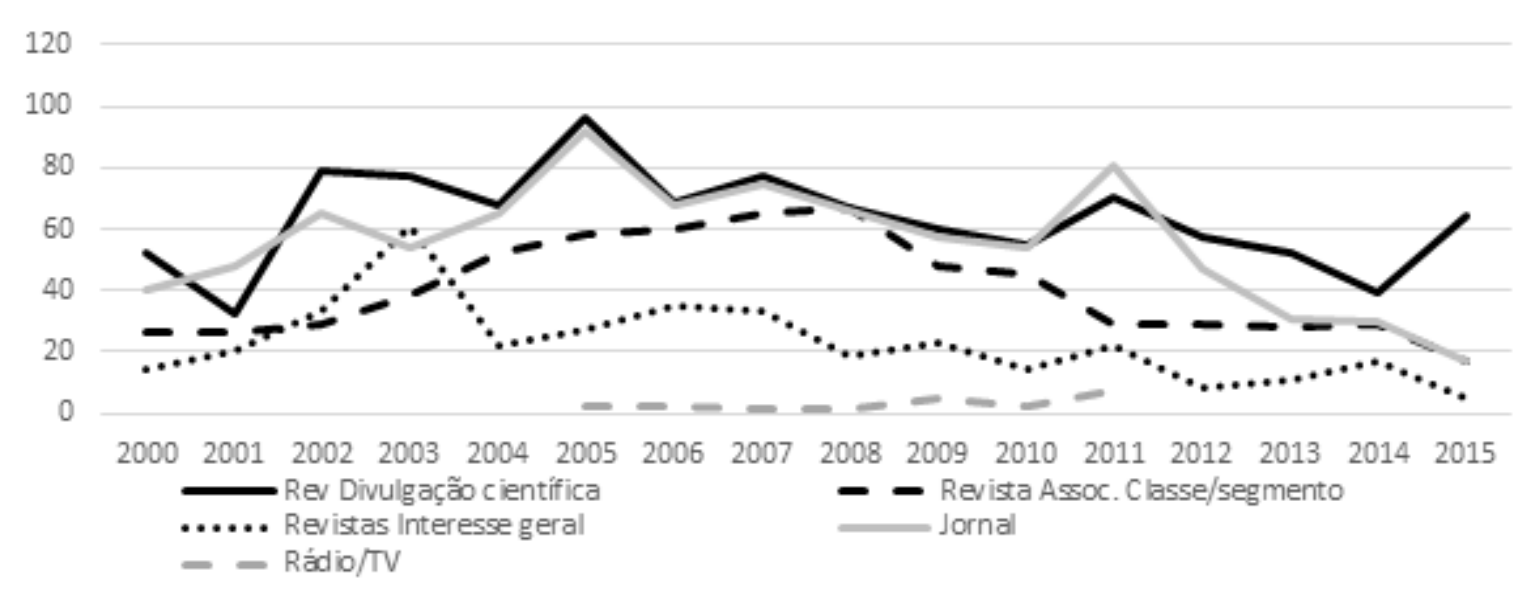

Fonte: Elaboração própria a partir dos dados coletados na plataforma Lattes 
As revistas de divulgação científica de 2000 até 2010 eram o tipo de veículo mais utilizado pelos pesquisadores, sendo ultrapassada pelos jornais somente em 2011, volta a ser sempre a com maior número de ocorrências, mesmo apresentando decréscimo ao longo dos anos. Em 2015 volta a ser crescente e o veículo mais utilizado, de acordo com a amostra analisada.

A categoria Revistas de Divulgação Científica reúne um total de 1.014 registros, distribuídos em 202 revistas diferentes. As 10 principais revistas, que contém 71\% da produção segundo o número de ocorrências, são listadas a seguir:

Tabela 3: Principais revistas de divulgação científica - produção em Jornais e Revistas 2000 a 2015

\section{TÍTULO DA PUBLICAÇÃO}

Science and Development Network

Ciência Hoje - Revista de divulgação científica da SBPC

RADIS - Comunicação em Saúde

Jornal da Ciência-SBPC

Agência Fiocruz de Notícias

WEBB

Pesquisa Fapesp

Amazonas faz Ciência FAPEAM

Círculo 21 - Portal de Opiniões (Jornal Eletrônico)

Agência Fapesp

Fiocruz - notícias
QUANT.

217

170

129

108

29

26

16

12

10

9

9

Fonte: elaboração própria a partir dos dados coletados na plataforma Lattes 
Apresenta-se, a seguir, um breve perfil das quatro principais revistas, onde 61,54\% da produção está registrada:

A Revista Science and Development Network (SciDev.Net) é uma publicação da organização internacional que leva o mesmo nome, fundada em 2001, com a proposta de divulgar ciência e tecnologia no Sul Global (América Latina e Caribe, Oeste médio e norte da África, Ásia do Pacífico e África Subsaariana), permitindo a republicação dos seus conteúdos sob as condições da Creative Commons Atribution Licence ${ }^{9}$. É uma publicação eletrônica, com escritório central baseado em Londres (UK), com representantes regionais para: América Latina e Caribe, Oriente Médio e Norte da África, Ásia e Pacífico e África Subsaariana ("Science \&amp; technology for global development news \&amp; analysis - SciDev.Net", [s.d.]).

A Revista Ciência Hoje, criada em 1982 pela SPBC hoje é uma das revistas publicadas pelo Instituto Ciência Hoje (ICH), uma organização privada, sem fins lucrativos, voltada à divulg ação científica no Brasil. Também é publicada pelo Instituto a revista Ciência Hoje das Crianças. Possui um corpo de editores científicos representantes das áreas de ciências humanas e sociais, ciências exatas e ciências biológicas, que valida o conteúdo de todas as suas publicações. É uma revista de periodicidade mensal, com versão impressa e digital ("Ciência Hoje | Site da Revista Ciência Hoje", [s.d.]).

A Revista RADIS, editada pela Escola Nacional de Saúde Pública (ENSP), da Fiocruz, foi lançada em 2002, fruto de um programa independente de jornalismo em saúde pública, iniciado em 1982. Os objetivos do projeto estavam nas iniciais do próprio nome: Reunião. Análise e Difusão de Informação sobre Saúde, Incluindo qualidade e condições de vida. Hoje a publicação é enviada para mais de 70 mil assinantes em vários municípios do país. Sua assinatura é gratuita ("Radis - comunicação em saúde”, [s.d.]).

0 Jornal da Ciência é uma publicação da SBPC, lançado em 1985, e desde 2014 passou a ser uma publicação digital como portal na internet, passando a ser produzido com o nome de JC Notícias. Uma edição mensal é impressa com conteúdo exclusivo e distribuída aos sócios ativos da SPBC e representantes das áreas de educação, ciência, tecnologia e inovação, sendo disponibilizada em versão digital no site da revista ("Jornal da Ciência”, [s.d.]).

9 https://creativecommons.org/ 


\section{CONSIDERAÇÕES FINAIS}

Este trabalho representa uma leitura inédita da comunicação de uma instituição de pesquisa para a sociedade, medida a partir dos dados registrados no currículo Lattes, que tem se mostrado uma rica fonte de pesquisa para os pesquisadores voltados a análises da produção em muitas áreas do conhecimento. Seu caráter autodeclaratório é uma limitação pois a fonte não tem recursos de curadoria. Como consequência, pode-se ter um mesmo registro constante em mais de uma categoria ou ser classificado de forma equivocada, como verificado com as 176 ocorrências que de fato são periódicos científicos.

Pelos resultados apresentados, conclui-se que ainda é bastante incipiente a divulgação científica, principalmente quando se compara a produção científica registrada na mesma fonte para o mesmo período, cujo total foi de 31.141 registros.

Por outro lado, conforme visto na página 5, a instituição toma pra si esta responsabilidade com muito vigor, haja visto a diversidade de canais descritos naquele item, tendo inclusive recebido em 20150 35 Prêmio José Reis de Divulgação Científica e Tecnológica, um reconhecimento a instituições, veículos ou pesquisadores que atuam na popularização da ciência, com intuito de levar a pesquisa para o público leigo ("Fiocruz ganha $35^{\circ}$ Prêmio José Reis de Divulgação Científica e Tecnológica”, [s.d.]).

Dentre os principais veículos utilizados pela comunidade de pesquisadores, vale ressaltar a produção disseminada por meio da Revista Ciência Hoje e o Jornal da Ciência, criados ainda na década de 1980. A primeira delas desempenha um papel real de divulgação para a sociedade, pois desde seu lançamento é vendida em bancas de jornais, ao passo que o segundo é voltado mais para disseminação entre os pares, mas pode ser acessado por qualquer cidadão com acesso à internet.

Ao promover a circulação de ideias e resultados de pesquisa, com a sociedade, a instituição exerce um papel fundamental para a reflexão sobre os impactos sociais e culturais das descobertas do cientista. A divulgação da ciência para a sociedade mostra-se uma ação fundamental para o próprio avanço da ciência.

\section{REFERÊNCIAS}

BUENO, W. C. Comunicação cientifica e divulgação científica: aproximações e rupturas conceituais. Informação \& Informação. Londrina, v. 15, n. supl, p. 1-12, 2010.

CANAL SAÚDE - FIOCRUZ. Disponível em: <http://www.canal.fiocruz.br/>. Acesso em: 23 jul. 2018. 
CANDOTTI, Ê. Ciência na educação popular. In: MASSARANI, L.; MOREIRA, I. C.; BRITO, F. (Eds.). . Divulgação e Jornalismo Científico em Saúde e Ambiente na Amazôia. Rio de Janeiro: [s.n.]. p. 15-23.

CIÊNCIA HOJE | Site da Revista Ciência Hoje. Disponível em: <http://cienciahoje.org.br/>. Acesso em: 23 nov. 2018.

FIOCRUZ - Pesquisa e ensino - SUS. Disponível em: <https://portal.fiocruz.br/pesquisa-e-ensino>. Acesso em: 23 jul. 2018.

FIOCRUZ GANHA 35 PRÊMIO JOSÉ REIS DE DIVULGAÇÃO CIENTÍFICA E TECNOLÓGICA. Disponível em: <http://www.museudavida.fiocruz.br/index.php/fiocruz-ganha-35-premio-jose-reis-de-divulgacaocientifica-e-tecnologica\#.XFw0D7jJ1PY>. Acesso em: 7 fev. 2019.

FONSECA, M. R. F. DA. As "Conferências Populares da Glória”: a divulgação do saber científico. História, Ciências, Saúde-Manguinhos, Rio de Janeiro, v. 2, n. 3, 1996.

GUIMARÃES, M. C. S. Comunicar a ciência: da divulgação científica ao engajamento em pesquisa. In: Divulgação e Jornalismo Científico em Saúde e Ambiente na Amazônia. Manaus: EDUA, 2014. p. 68-78.

JORNAL DA CIÊNCIA. Disponível em: <http://www.jornaldaciencia.org.br/>. Acesso em: 23 nov. 2018.

MASSARANI, L.; MOREIRA, I. DE C.; BRITO, F. Ciência e público: caminhos da divulgação científica no Brasil. Rio de Janeiro: Casa da Ciência - UFRJ, 2002. v. 1

0 QUE É 0 MUSEU DA VIDA? Disponível em: <http://www.museudavida.fiocruz.br/index.php/sobre-0museu/o-que-e-o-museu-da-vida>. Acesso em: 23 jul. 2018.

PLATAFORMA LATTES CNPQ - SOBRE. Disponível em: <http://lattes.cnpq.br/>. Acesso em: 29 jan. 2019.

RÁDIO SOCIEDADE. Disponível em: <http://www.fiocruz.br/radiosociedade/cgi/cgilua.exe/sys/start. htm?sid=2>. Acesso em: 22 jul. 2018.

RADIS - COMUNICAÇÃO EM SAÚDE. Disponível em: <http://www6.ensp.fiocruz.br/radis/>. Acesso em: 23 jul. 2018. 
SCIENCE \&AMP; technology for global development news \&amp; analysis - SciDev.Net. Disponível em: <https://www.scidev.net/global/>. Acesso em: 23 nov. 2018.

VIDEOSAÚDE - Distribuidora da Fiocruz | ICICT | Fiocruz. Disponível em: <https://www.icict.fiocruz.br/ content/videosaude-distribuidora-da-fiocruz-1>. Acesso em: 23 jul. 2018. 